\title{
Unscarred uterine rupture and subsequent pregnancy outcome - a tertiary centre experience
}

\author{
Nurullah Peker ${ }^{1}$, Edip Aydın² ${ }^{(D}$, Mehmet Sıddık Evsen ${ }^{1}\left(\mathbb{D}\right.$, Fatma Nur Hançer $^{2}{ }^{(D)}$,

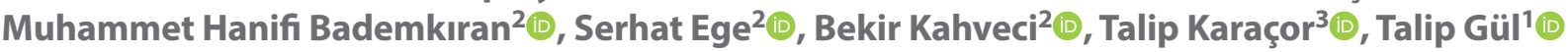 \\ ${ }^{1}$ Department of Obstetrics and Gynecology, Dicle University, Faculty of Medicine, Diyarbakır, Turkey \\ ${ }^{2}$ Department of Obstetrics and Gynecology, Health Sciences University, Gazi Yasargil Training and Research Hospital, Diyarbakir, Turkey \\ ${ }^{3}$ Department of Obstetrics and Gynecology, Adıyaman University, Faculty of Medicine, Adıyaman, Turkey
}

\begin{abstract}
Objectives: The aim of this study was to investigate the incidence, etiology and obstetric outcomes of rupture in unscarred uterine rupture and in those with a history of uterine rupture

Material and methods: The hospital records of women who had delivered between May 2005 and May 2017 at a tertiary center were examined retrospectively. Data on patients with unscarred uterine rupture in pregnancy who had undergone fertility-preserving surgery were evaluated.

Results: During the study period, 185,609 deliveries occurred. Of those, unscarred uterine rupture has occurred in 67 women. There were no ruptures reported in nulliparous women. The rupture was observed in the isthmic region in $60(89.6 \%)$ patients and in the fundus in $7(10.4 \%)$ patients. Thirty-eight (56.7\%) patients had undergone a total or subtotal hysterectomy, and 29 (43.3\%) patients had received primary repair. Ten patients had reconceived after the repair. Of these, eight patients who had a history of isthmic rupture, successfully delivered by elective C-section at 36-37 wk. of gestation, and two experienced recurrent rupture at 33 and $34 \mathrm{wk}$. of gestation, respectively. Both patients had a history of fundal rupture, and their inter-pregnancy interval was 9 and 11 mo., respectively.

Conclusions: The incidence of rupture in unscarred pregnant uteri was found to be one per 2,770 deliveries. Owing to the high morbidity, regarding more than half of the cases with rupture eventuated in hysterectomy, clinicians should be prudent in induction of labour for multiparous women since it was the main cause of rupture in this series. Short inter-pregnancy intervals and history of fundal rupture may confer a risk for rupture recurrence. Those risk factors for recurrence should be validated in another studies.
\end{abstract}

Key words: unscarred uterine rupture; recurrent uterine rupture; uterine repair; timing of delivery; pregnancy outcome

Ginekologia Polska 2020; 91, 2: 95-99

\section{INTRODUCTION}

Unscarred uterine rupture (UUR) in pregnancy is associated with a high risk of maternal and neonatal mortality and morbidity rates [1]. Although it is a rare event, the overall rate of UUR ranges from $1 / 5700$ to $1 / 20,000$ pregnancies [2-5]. Rupture can be caused by traumas, including abdominal trauma and labour induction, or it can occur spontaneously and be associated with grand multiparity, malpresentation, placental invasion and prolonged labour $[6,7]$.

In UUR cases, the goals of surgery is to control the haemorrhage, to identify injury of other intra-abdominal organs (urinary tract, etc.) and to minimize early post-surgical morbidity. The ruptured uterus must either be removed or repaired. The decision to perform a hysterectomy is made according to a combination of factors, including the patient's desire for future fertility, the extent of the uterine injury, intra-operative haemodynamics and anaesthetic stability and the skill of the surgeon in complicated UUR cases $[8,9]$.

There is no consensus in the literature on the optimal timing of conception and timing of elective cesarean delivery in patients with a history of UUR who underwent uterus-preserving surgery in the past. The aim of this study was to investigate obstetric outcomes of UUR and optimal timing of elective cesarean delivery and recurrence of uterine rupture (UR) in patients with a history of UUR. 


\section{MATERIAL AND METHODS}

The hospital records of women who had delivered between May 2005 and May 2017 at the obstetrics clinic of Diyarbakir Health Sciences University, Gazi Yasargil Training and Research Hospital were examined retrospectively. Prior to the study, approval was obtained from the local ethics committee of Dicle University School of Medicine (ethics committee approval no. 40).

UUR is defined as a full thickness separation of uterine wall and the overlying serosa. Incomplete UR cases with serosa intact, patients who had a history of previous cesarean delivery, and patients diagnosed with rupture in an external centre and referred to our clinic were excluded. UUR was diagnosed with high suspicion index including rapid fetal heart rate changes, acute or constant abdominal pain, uterine tenderness and loss of station and confirmed intraoperatively as extracted from the medical records.

Data on the age, parity number, birth weight, gestational week, cause and site of rupture, duration of surgery, length of hospital stay, amount of erythrocyte suspensions transfused, maternal and perinatal mortality and treatment modalities were noted.

In some patients, Oxytocin 1-2 $\mathrm{mlU} / \mathrm{min}$ was used as the beginning dose for labour induction and augmentation. In some of these patients, it was detected that oxytocin was given more than the determined dose. As a result, hyperstimulation findings were seen in the non-stress test. This situation was defined as uncontrolled labour induction. The mismatch between the size of the fetal head and the maternal pelvis in the vaginal examination and as a result the failure to progress in labour was defined as cephalopelvic disproportion. Mothers over 35 years of age were taken as advanced maternal age.

Total or subtotal hysterectomy was performed in patients with unstable hemodynamics. Uterus-preserving surgery was performed in patients with stable hemodynamics and desire for future fertility. In uterus-preserving surgery, the rupture area was repaired primary.

\begin{tabular}{|l|l|l|}
\hline \multicolumn{3}{|c|}{ Table 1. Demographic, obstetric and clinical data of the patients } \\
\hline Characteristics & Mean \pm SD & Min-Max \\
\hline Age (y) & $35.28 \pm 6.83$ & $18-47$ \\
\hline Parity & $5.45 \pm 2.75$ & $1-13$ \\
\hline Birth weight (g) & $3,344 \pm 953$ & $480-4750$ \\
\hline Gestational week & $38.01 \pm 4.20$ & $21-42$ \\
\hline Hospitalization duration (d) & $4.9 \pm 2.1$ & $1-11$ \\
\hline Duration of surgery (min) & $97.8 \pm 39.9$ & $60-240$ \\
\hline Amount of transfusion (unit) & $4.1 \pm 2.5$ & $0-13$ \\
\hline
\end{tabular}

SD — standard deviation
Data on patients who had undergone fertility-preserving surgery were accessed, and their subsequent pregnancy status, the timing of delivery and occurrence of recurrent ruptures were recorded after obtaining informed patients consent.

\section{Statistical analysis}

Data were analysed using SPSS 22 for Windows (SPSS Inc., Chicago, IL, USA). The normal distribution of the data was tested using the Shapiro-Wilk test. Data are expressed as mean \pm standard deviation and minimum, maximum and percentage, as appropriate.

\section{RESULTS}

Of 185,609 delivery during the study period, 67 of them culminated by UUR. Accordingly, the incidence was found to be as one in 2,770 pregnancies. Demographic, obstetric and clinical data of the patients with UUR are shown in Table 1 and 2.

Twenty (30\%) pregnant women with UUR were admitted with a completely dilated cervix and taken to the delivery room for an emergency C-section. These patients had not been appropriately followed up during the antenatal period. Furthermore, they had been admitted after unsuccessful attempts at in home-deliveries with the help of a local midwife. Upon admission, there was no foetal heart beat in 16 of these 20 patients. Among these patients, three had foetuses with advanced hydrocephalus, two had a transversely located foetus with a vaginally prolapsed arm, and one had a breech presentation.

Thirty-eight (56.7\%) patients had undergone a total or subtotal hysterectomy and 29 (43.3\%) patients had received primary repair. Twelve patients had additional sal-

\begin{tabular}{|c|c|c|}
\hline Characteristics & & $\mathbf{n}$ \\
\hline \multirow[t]{2}{*}{ Age } & $<35$ & $31(46.3 \%)$ \\
\hline & $\geq 35$ & $36(53.7 \%)$ \\
\hline \multirow[t]{2}{*}{ Parity } & $<5$ & $26(38.8 \%)$ \\
\hline & $\geq 5$ & $41(61.1 \%)$ \\
\hline \multirow[t]{2}{*}{ Site of rupture } & Isthmic region & $60(89.6 \%)$ \\
\hline & Fundus & $7(10.4 \%)$ \\
\hline \multirow[t]{2}{*}{ Induction of labor } & Yes & $45(67.2 \%)$ \\
\hline & No & $22(32.8 \%)$ \\
\hline \multirow[t]{3}{*}{ Cause of rupture } & Uncontrolled labour induction & $32(47.8 \%)$ \\
\hline & Cephalopelvic disproportion & $26(38.8 \%)$ \\
\hline & Malpresentation & $9(13.4 \%)$ \\
\hline \multirow[t]{2}{*}{ Type of surgery } & Total or subtotal hysterectomy & $38(56.7 \%)$ \\
\hline & Primary repair & $29(43.3 \%)$ \\
\hline
\end{tabular}


pingo-oophorectomy due to UR with adnexal involvement and 25 patients had required hypogastric arterial ligation. A repair procedure and bilateral tubal ligation had been performed in two patients. The adverse events were as follows: intra-operative bladder injury $(n=5)$; absence of a foetal heart beat $(n=21)$, new-born deaths $(n=2)$ and maternal deaths due to an uncontrolled haemorrhage $(n=2)$.

Ten of the 29 patients had reconceived after UR repair. Of these, eight patients who had a history of isthmic rupture delivered by an elective C-section at 36-37 wk. of gestation with no complication, and the mean inter-pregnancy interval of the remaining eight patients was $36 \mathrm{mo}$. (range: 24-48 mo.). The other two patients experienced recurrent ruptures at 33 and 34 wk of gestation, respectively. These two patients had a history of fundal rupture, and their inter-pregnancy interval was 9 and 11 mo., respectively. Figure 1 shows the conception status of the 29 patients following uterine repair.

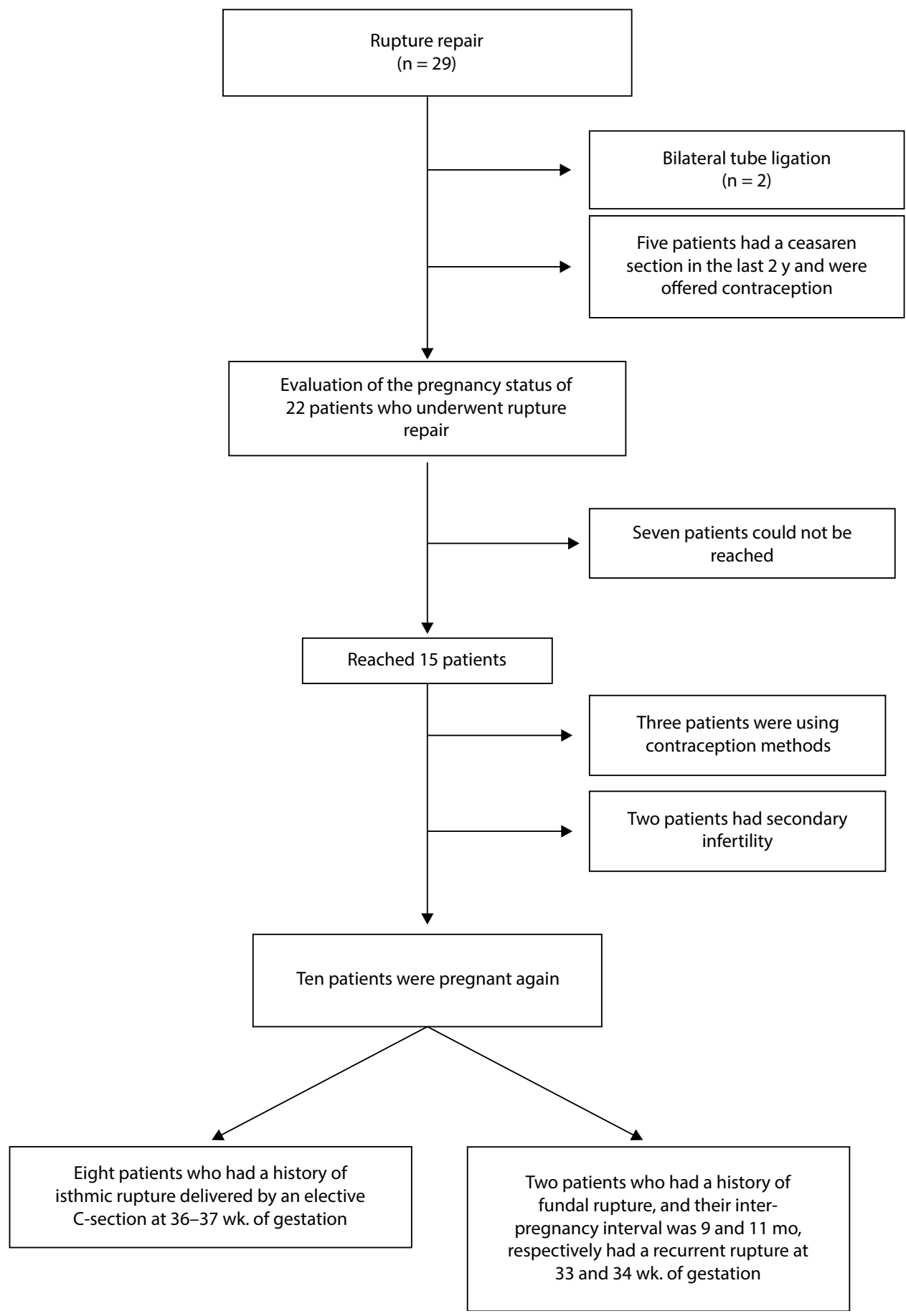

Figure 1. Conception status of the patients after uterine repair 


\section{DISCUSSION}

In this study, clinical findings of UUR, and subsequent pregnancy outcomes of these patients who underwent uterine preserving surgery were examined. It was observed that in most of the patients' rupture occurred in the isthmic region and hysterectomy was performed in many of those. The patients who underwent uterus-preserving surgery with primary repair were evaluated for their next pregnancy. It was found that the patients with UUR from the isthmic region had a caesarean section without complications, and the patients with UUR from the fundus developed recurrent rupture again in the early weeks.

Previous studies reported that UUR occurred in one in every 5,700-20.000 pregnancies [2-5]. In the present study, the incidence of UR not associated with previous uterine surgery was $1 / 2770$. This figure seems to be higher than that reported in the literature. Advanced maternal age and grand multiparity, both of which are common in southeast Turkey may have contributed to the increased incidence of UR. In addition, 20 of the patients who presented to our clinics, a referral centre for the region, with UR did so only after attempting to deliver at home, traditionally. This may have been a contributory factor to the high incidence of UR in this study.

According to the literature, the risk factors for UUR are as follows: high parity, use of uterotonic drugs, advanced maternal age, dystocia, macrosomia and possibly short inter-pregnancy intervals $[1,7,8,10]$. In agreement with the literature, the causes of the UUR's in the present study included advanced age, grand multiparity, uncontrolled labour induction, cephalopelvic disproportion and malpresentation. In addition, in several patients, more than one risk factor was responsible for UR, as was reported in a previous study [11]. The mean gestational age was $38.01 \mathrm{wk}$. in the present study, and the patients presented to the clinic during labour or in late pregnancy. These findings are in accordance with those in the literature [12].

Previous studies reported that the isthmic region was the most common site of UR $[13,14]$. Similarly, the isthmic region was the most common site of UR in the present study. This situation is attributable to the cervix becoming thinner during labour and besides, accommodating isthmic region having fewer contractile cells as compared with that of the upper uterine region [15].

UUR is reported to cause several maternal complications and sequelae, such as severe haemorrhages, bladder lacerations, hysterectomies and mortality [16-18]. Also previous studies pointed to an association between UR and foetal mortality and morbidity $[16,19]$. Similarly, UR culminated in adverse maternal and neonatal outcomes in the present study. In terms of intra-operative complications, five patients experienced bladder injuries, and several patients required a blood transfusion because of a severe haemorrhage. An uncontrolled haemorrhage was responsible for mortality in two patients, both of whom were advanced aged grand multiparous patients who presented to the hospital during labour. In the present study, 21 foetuses had no heart beat at the time of maternal presentation to the clinic, and two foetuses died in the post-natal period.

A hysterectomy, whether total or subtotal, is the main surgical procedure adopted in cases of UR. Primary repair is an option for patients desiring future fertility [14]. In this study, the most frequently adopted surgical procedure was a total or subtotal hysterectomy because of the advanced maternal age, grand multiparity and accordingly a lack of desire for future pregnancy and the severity of uterine injury. Hysterectomy at full dilatation can be a challenging procedure [20]. UUR with a completely dilated cervix and subsequently underwent to emergency C-section was observed in $30 \%$ of women with UUR in the current study. On the other hand, 29 (43.3\%) younger patients with a desire for future fertility underwent uterine repair. Twenty-five patients also required hypogastric arterial ligation for the management of a haemorrhage. In such cases, it should be kept in mind that hypogastric arterial ligation may help to achieve haemostasis [21].

According to the literature, the risk of recurrent UR is linked to the site of rupture as the highest risk is linked to fundal injuries [22]. Previous studies reported the risk of recurrent rupture as $22-100 \%$ in patients who reconceived after uterine repair $[23,24]$. In a study on five patients with UR, Lim et al. [23] reported that all the patients delivered by a $\mathrm{C}$-section, with no recurrent ruptures. However, the small size of their study population means it is not possible to draw robust conclusions. A previous study recommended an inter-pregnancy interval of at least 18 mo. for women who desired fertility following the repair of an unscarred uterus that had ruptured during pregnancy [25]. In the present study, both patients in whom UR recurred had a short inter-pregnancy period. We agree that a short inter-pregnancy interval may be a major risk factor for recurrent ruptures. Chibber et al. [24] reported that 24 of 44 rupture cases had undergone a repair procedure and that 22 of these 24 cases had reconceived. Furthermore, 20 of these 22 cases delivered by a planned C-section, with no maternal or foetal complications. The remaining two cases had no proper follow up during the antenatal period and experienced recurrent UR at 32 and 35 wk. of gestation, respectively. However, no information has been given as to where these two patients had ruptures in their previous pregnancy. In this study, there were no complications in patients with a previous history of fundal rupture in subsequent pregnancies. However, it was determined that both patients who had ruptured from the isthmic region developed recurrent rupture in early gestational weeks. 
This study has some limitations, primarily that it is retrospective study, which could be open to selection bias. Likewise, it lacks data on body mass index, diabetes mellitus, and uterine pathologies (i.e., myoma uteri). In addition, that various surgeons performed the operations and might limit the real complication rate because there was no specific information regarding the surgeons' levels of experience. Finally, seven patients who could not be reached after UUR repair were seen as deficiency in our results. The strengths of the study include a very broad cohort of evaluated cases despite being a single-center study. Further, the evaluation of the next pregnancy outcomes of patients who underwent uterus-preserving surgery makes the study powerful.

There is no consensus in the literature on the optimal timing of conception and timing of elective cesarean delivery in patients with a history of UUR who underwent uterus-preserving surgery in the past. The timing of delivery of a subsequent pregnancy in UUR cases should be considered carefully, as recurrent rupture may occur early in the third trimester. Obstetricians might consider reducing the risk of recurrent ruptures by recommending a $\mathrm{C}$-section and scheduling the delivery before the onset of labour. Authors postulate that a C-section at 36-37 wk. of gestation may be recommended for patients with a history of isthmic ruptures. To avoid potential early recurrent rupture, an earlier C-section at 32-33 wk. of gestation after completion of foetal lung maturation may be recommended for patients with a history of fundal ruptures and those with short inter-pregnancy intervals. These recommendations should be cautiously approached due to a current lack of data and more research is urgently needed.

\section{CONCLUSIONS}

UUR constitutes a major risk factor for fetomaternal morbidity and mortality. The incidence of rupture in unscarred pregnant uteri was found to be one per 2,770 deliveries. Owing to the high morbidity regarding more than half of the cases with rupture eventuated in hysterectomy, clinicians should be prudent in induction of labour for multiparous women since it was the main cause of rupture in this series.

\section{Acknowledgements}

We would like to thank Ayhan Aktaş his assistance with the statistics used in this report.

\section{REFERENCES}

1. Gibbins $\mathrm{K}$, Weber T, Holmgren $\mathrm{C}$, et al. Maternal and fetal morbidity associated with uterine rupture of the unscarred uterus. American Journal of Obstetrics and Gynecology. 2015; 213(3): 382.e1-382.e6, doi: 10.1016/j. ajog.2015.05.048.

2. Dow M, Wax J, Pinette $M$, et al. Third-Trimester Uterine Rupture without Previous Cesarean: A Case Series and Review of the Literature. American Journal of Perinatology. 2009; 26(10): 739-744, doi: 10.1055/s-0029-1223287.

3. Porreco $R$, Clark $S$, Belfort $M$, et al. The changing specter of uterine rupture. American Journal of Obstetrics and Gynecology. 2009; 200(3): 269.e1-269.e4, doi: 10.1016/j.ajog.2008.09.874.
4. Miller DA, Goodwin TM, Gherman RB, et al. Intrapartum rupture of the unscarred uterus. Obstet Gynecol. 1997; 89(5 Pt 1): 671-673, doi: 10.1016/s0029-7844(97)00073-2, indexed in Pubmed: 9166298.

5. Zwart JJ, Richters JM, Öry F, et al. Uterine rupture in the Netherlands: a nationwide population-based cohort study. BJOG: An International Journal of Obstetrics \& Gynaecology. 2009; 116(8): 1069-1080, doi: 10.1111/j.1471-0528.2009.02136.x.

6. You SH, Chang YL, Yen CF. Rupture of the scarred and unscarred gravid uterus: Outcomes and risk factors analysis. Taiwanese Journal of Obstetrics and Gynecology. 2018; 57(2): 248-254, doi: 10.1016/j. tjog.2018.02.014.

7. Pierzynski P, Laudanski P, Lemancewicz $A$, et al. Spontaneous rupture of unscarred uterus in the early second trimester: a case report of placenta percreta. Ginekol Pol. 2012; 83(8): 626-629, indexed in Pubmed: 23342889.

8. Walsh C, Baxi L. Rupture of the Primigravid Uterus: A Review of the Literature. Obstetrical \& Gynecological Survey. 2007; 62(5): 327-334, doi: 10.1097/01.ogx.0000261643.11301.56.

9. Kapoor DS, Sharma SS, Alfirevic Z. Management of unscarred ruptured uterus. Journal of Perinatal Medicine. 2003; 31(4), doi: 10.1515/jpm.2003.048.

10. Wielgos M, Bomba-Opoń D, Breborowicz G, et al. Recommendations of the Polish Society of Gynecologists and Obstetricians regarding caesarean sections. Ginekologia Polska. 2018; 89(11): 644-657, doi: 10.5603/gp.a2018.0110.

11. Ofir K, Sheiner E, Levy A, et al. Uterine rupture: differences between a scarred and an unscarred uterus. American Journal of Obstetrics and Gynecology. 2004; 191(2): 425-429, doi: 10.1016/j.ajog.2004.01.026.

12. Wang $\mathrm{YL}$, Su TH. Obstetric Uterine Rupture of the Unscarred Uterus: A Twenty-Year Clinical Analysis. Gynecologic and Obstetric Investigation. 2006; 62(3): 131-135, doi: 10.1159/000093031.

13. Konje JC, Odukoya OA, Ladipo OA. Ruptured uterus in Ibadan - A twelve year review. International Journal of Gynecology \& Obstetrics. 2004; 32(3): 207-213, doi: 10.1016/0020-7292(90)90347-n.

14. Turgut A, Ozler A, Evsen $M$, et al. Uterine rupture revisited: Predisposing factors, clinical features, management and outcomes from a tertiary care center in Turkey. Pakistan Journal of Medical Sciences. 2013; 29(3), doi: 10.12669/pjms.293.3625.

15. William???s Obstetrics. Academic Medicine. 1936; 11(5): 336, doi: 10.1097/00001888-193609000-00027.

16. Chauhan S, Martin J, Henrichs C, et al. Maternal and perinatal complications with uterine rupture in 142,075 patients who attempted vaginal birth after cesarean delivery: A review of the literature. American Journal of Obstetrics and Gynecology. 2003; 189(2): 408-417, doi: 10.1067/s0002-9378(03)00675-6.

17. Kwee $A$, Bots $M$, Visser $G$, et al. Uterine rupture and its complications in the Netherlands: A prospective study. European Journal of Obstetrics \& Gynecology and Reproductive Biology. 2006; 128(1-2): 257-261, doi: 10.1016/j.ejogrb.2006.02.005.

18. Zheng J, Liu S, Xing J. Prognosis and related risk factors of patients with scarred uterus complicated with central placenta previa. Ginekologia Polska. 2019; 90(4): 185-188, doi: 10.5603/gp.2019.0033.

19. Phelan J, Korst L, Martin G. Causation-Fetal Brain Injury and Uterine Rupture. Clinics in Perinatology. 2007; 34(3): 409-438, doi: 10.1016/j. clp.2007.03.014.

20. Dogan O, Pulatoglu C, Yassa M. A new facilitating technique for postpartum hysterectomy at full dilatation: Cervical clamp. Journal of the Chinese Medical Association. 2018;81(4):366-369, doi: 10.1016/j.jcma.2017.05.010.

21. Camuzcuoglu $\mathrm{H}$, Toy $\mathrm{H}$, Vural $\mathrm{M}$, et al. Internal iliac artery ligation for severe postpartum hemorrhage and severe hemorrhage after postpartum hysterectomy. Journal of Obstetrics and Gynaecology Research. 2010; 36(3): 538-543, doi: 10.1111/j.1447-0756.2010.01198.x.

22. Usta I, Hamdi M, Musa AA, et al. Pregnancy outcome in patients with previous uterine rupture. Acta Obstetricia et Gynecologica Scandinavica. 2007; 86(2): 172-176, doi: 10.1080/00016340601089768.

23. Lim A, Kwee A, Bruinse H. Pregnancy After Uterine Rupture: A Report of 5 Cases and a Review of the Literature. Obstetrical \& Gynecological Survey. 2005; 60(9):613-617, doi: 10.1097/01.ogx.0000176677.26657.6c.

24. Chibber $\mathrm{R}$, El-Saleh $\mathrm{E}$, Fadhli $\mathrm{R}$, et al. Uterine rupture and subsequent pregnancy outcome - how safe is it? A 25-year study. The Journal of Maternal-Fetal \& Neonatal Medicine. 2010; 23(5): 421-424, doi: 10.3109/14767050903440489.

25. Bujold E, Gauthier R. Risk of Uterine Rupture Associated With an Interdelivery Interval Between 18 and 24 Months. Obstetrics \& Gynecology. 2010; 115(5): 1003-1006, doi: 10.1097/aog.0b013e3181d992fb. 